\title{
On the Question of Nonlinear Fluctuations of Heavy Ropes
}

\author{
SERGEY O. GLADKOV \\ Moscow Aviation Institute (National Research University) (MAI) \\ Volokolamskoe Shosse, 4. \\ 125993, Moscow \\ RUSSIA
}

Abstract: - Due to the methods of the dynamics of curvilinear motion the system of nonlinear differential equations describing two-dimensional arbitrary displacements of a heavy rope, one end of which is free, and the other is fastened is obtained. The problem is solved in the language of displacement vector $\vec{\xi}(x, t)=\xi_{x}(x, t) \mathbf{i}+\xi_{y}(x, t) \mathbf{j}$, where $\xi_{x}(x, t)$ and $\xi_{y}(x, t)$ accordingly, arbitrary offsets of the rope points in the axes of coordinates. It is shown that in the limit of small oscillations the obtained equations are reduced to the description of the weak offsets of the horizontal string fixed on both ends, and small displacements of the heavy vertical filament, fluctuating due to the force of gravity. The main goal of the paper is the mathematical description of the strong non-equilibrium dynamic systems.

Keywords: - Offsets, heavy rope, gravity, nonlinear differential equations.

Received: September 24, 2019. Revised: February 12, 2020. Re-revised: March 10, 2020.

Accepted: March 18, 2020. Published: March 20, 2020.

\section{Introduction}

In recent decades, a variety of nonlinear problems have become very popular, dedicated to the description of a lot of real physical, chemical and biological processes flowing in gases, liquids and solids in different model conditions. The overwhelming majority of the set tasks are solved due to methods of numerical integration of nonlinear differential equations. in this case, a number of interesting features can be identified and a remarkable example here is the classic Lorentz solution, describing the hydrodynamics of the flatparallel flow of fluid between two parallel horizontal planes at convection accounting. Note, by the way, that at numerical decision of the received system of equations Lorentz the strange attractor has been found. Unfortunately, the attractor is not a property of any nonlinear system of equations, and its detection is always accompanied by the undisguised joy of the researcher who discovered it.

In our work we will talk about the derivation of the system of nonlinear dynamic equations in the conditions of arbitrary fluctuations of the heavy rope, deduced from the equilibrium position, for example, by air flow or as a result of simple dynamic deviation from Equilibrium positions. The solution of the task is purely analytical, and we will test the obtained equations due to the limit transitions to various classical cases, from which some interesting regularities that are not previously mentioned in any of the publications known to us, devoted to analysis of solutions of different types of equations in private derivatives (for example, see monographs [1], [2], as well as a lot of purely mathematical articles on the theory of mathematical physics equations). Some estimations and calculations have been preliminary made by us in work [3] where the movement of a rope in a viscous continuum taking into account local force of resistance is investigated. We will not consider energy dissipation in this work. The article includes Introduction, The Main Part, analysis of the resulting equations, basic results, conclusion and list of used literature.

The main merit of the work is the conclusion of a system of non-linear differential equations describing arbitrary fluctuations of heavy ropes. In private cases, these equations are transferred to known equations, which indicates their correctness. This is now the first attempt to produce equations that describe the complex dynamic behavior of long - dimension objects. The results of the article can be compared to the private results obtained in the work [3].

\section{Decision of the Problem}

Imagine a rope suspended in one end (see Figure 1), and put before itself the task to describe its shape and dynamics of movement at any time after removing it from the position of equilibrium. The free end of the rope is rejected in a co mpletely arbitrary manner. This is the initial deviation or otherwise, the initial form of the rope, we will ask how 


$$
\xi(x, 0)=\mathbf{S}(x)
$$

According to the geometry of fig. 1, where $\mathbf{v}$ Is the speed of moving the surface points of the rope, for which $\mathbf{v} \boldsymbol{\tau}=\mathrm{V}$, where $\boldsymbol{\tau}-$ is single vector tangent, held in an arbitrary point of the rope, angle $\alpha-$ is outer angle between tangent and axis $x$.

For the conclusion of the basic equations of the rope motion we will write its full energy in the form of kinetic $T$ and potential energies $U$, namely

$$
E=T+U=\frac{1}{2} \int_{l} \rho \dot{\xi}^{2} d l+\int_{l} u d l+\int_{l} \rho \mathbf{g} \cdot \xi d l,
$$

where $\rho-$ is linear density of the rope, $\dot{\xi}=\frac{\partial \xi}{\partial t}-$ is speed of each element of the rope, $u-$ is the potential energy attributable to the unit length of the rope, $\mathbf{g}-$ is the acceleration of gravity and $l-$ is the rope length.

Here it is necessary to pay special attention to the physical sense of potential energy $u$ that is the unit length of the rope. The value $\frac{u}{\rho}$ has dimension squares speed. This speed characterizes the speed of moving the surface points of the rope, and in the limit of small movements of the rope (or string) becomes equal to the speed of its longitudinal oscillations $\mathrm{v}_{0}$. It is obvious that in general it should be considered that

$$
\mathrm{v}=\sqrt{\frac{u}{\rho}} .
$$

It is quite clear that the velocity vector of the surface points is defined as $\mathbf{v} \boldsymbol{\tau}=\mathbf{V}$. For this speed we have the right to immediately write equations of motion, which will be quite similar to the equations, strictly deduced by us in works [4] - [7]. Indeed, since it is a matter of free fluctuations, each point of the rope will have to move along its trajectory at some speed $\mathbf{u}$ perpendicular to the speed $\mathbf{v}$ (see Figure 1), which allows us to write the following system of equations immediately taking into account the results of the work [4]

$$
\left\{\begin{array}{l}
\dot{u}=\frac{\mathrm{v}^{2}}{R_{1}}+g \sin \alpha_{1}-k_{1} \frac{u}{\rho}, \\
\frac{u^{2}}{R_{2}}=g \cos \alpha_{1}-\dot{v}+\frac{f}{\rho},
\end{array},\right.
$$

where $R_{1}$ - is radius of curvature at a given point in the rope (see Figure 1), $R_{2}-$ is radius of curvature of the trajectory, which moves the arbitrary point of the rope, and $R_{1} \perp R_{2}$ at each point in time and at each point of the rope, $f-$ is the linear density of force caused by the internal tense state of the rope, $k_{1}-$ is the coefficient of resistance of the continuum in which the rope moves, $\rho-$ is linear density of the rope. Corners $\alpha_{1}$ and $\alpha_{2}$ shown in the Figure 1 .

The speed of moving the rope points (see work [4]) can be determined as

$$
\mathrm{v}=-R_{1} \dot{\alpha}_{1}
$$

where the radius of curvature at an arbitrary point of the rope for the two-dimensional case we are in is

$$
\frac{1}{R_{1}}=\frac{\frac{d^{2} \xi_{y}}{d \xi_{x}^{2}}}{\left(1+\left(\frac{d \xi_{y}}{d \xi_{x}}\right)^{2}\right)^{\frac{3}{2}}}
$$

We emphasize that all derivatives in (7) are taken for a fixed point of time $t$. In the language of corner coefficient $k_{1}=\frac{d \xi_{y}}{d \xi_{x}}$ the expression (6) can be represented as an equivalent

$$
\frac{1}{R_{1}}=\frac{\frac{d k_{1}}{d \xi_{x}}}{\left(1+k_{1}^{2}\right)^{\frac{3}{2}}} .
$$

As to the radius of curvature $R_{2}$, it can be represented in the form similar to formulas (6) and (7), but recorded already through some new independent function $\psi=\psi\left(\xi_{x}\right)$ that is 


$$
\frac{1}{R_{2}}=\frac{\frac{d^{2} \psi}{d \xi_{x}^{2}}}{\left(1+\left(\frac{d \psi}{d \xi_{x}}\right)^{2}\right)^{\frac{3}{2}}}
$$

or using a corner coefficient $k_{2}=\frac{d \psi}{d \xi_{x}}$ in the form of

$$
\frac{1}{R_{2}}=\frac{\frac{d k_{2}}{d \xi_{x}}}{\left(1+k_{2}^{2}\right)^{\frac{3}{2}}} .
$$

The condition of transversality (see [9] - [17]) for an arbitrary point of the rope we can imagine how

$$
\left.k_{1} k_{2}\right|_{\substack{t t t\left(\xi_{x}, \xi_{y}\right) \\ x=x\left(\xi_{x}, \xi_{y}\right)}}=-1
$$

Thus, the system of equations (4) is completely defined. Now we have to find only equations for the offset functions $\xi_{x}$ and $\xi_{y}$.

In our opinion, the most convenient and rational method of withdrawal of any dynamic equations of motion is the use of the law of conservation of energy (see, for example, work [8]). In fact, it is very convenient to use the following approach as part of the task. Because the rope length element is

$d l=\sqrt{\xi_{x}^{\prime 2}+\xi_{y}^{\prime 2}} d x$,

by differentiating total energy (2) by t ime, and considering that the upper limit of integration is also a function of time, we find as a result

$$
\begin{aligned}
& 2 \dot{E}=\frac{\partial}{\partial t} \int_{x_{0}}^{x_{1}} \rho\left(\dot{\xi}^{2}+2 \mathrm{v}^{2}+2 \mathbf{g} \cdot \xi\right) \sqrt{\xi_{x}^{\prime 2}+\xi_{y}^{\prime 2}} d x= \\
& =\int_{x_{0}}^{x_{1}} \rho\left(\dot{\xi}^{2}+2 \mathrm{v}^{2}+2 \mathbf{g} \cdot \xi\right) \frac{\left(\xi_{x}^{\prime} \dot{\xi}_{x}^{\prime}+\xi_{y}^{\prime} \dot{\xi}_{y}^{\prime}\right)}{\sqrt{\xi_{x}^{\prime 2}+\xi_{y}^{\prime 2}}} d x+ \\
& +\int_{x_{0}}^{x_{1}} \rho(2 \dot{\xi} \cdot \ddot{\xi}+2 \mathbf{g} \cdot \dot{\xi}) \sqrt{\xi_{x}^{\prime 2}+\xi_{y}^{\prime 2}} d x+ \\
& +\left.\dot{x}_{1} \rho\left(\dot{\xi}^{2}+2 \mathrm{v}^{2}+2 \mathbf{g} \cdot \xi\right) \sqrt{\xi_{x}^{\prime 2}+\xi_{y}^{\prime 2}}\right|_{x=x_{1}}=0 .
\end{aligned}
$$

The first term in (12) is integrated by parts, allocating private derivatives by time $\dot{\xi}_{x}$ and $\dot{\xi}_{y}$. As a result we find

$$
\begin{aligned}
& 2 \dot{E}=\frac{\partial}{\partial t} \int_{x_{0}}^{x_{1}} \rho\left(\dot{\xi}^{2}+2 \mathrm{v}_{0}^{2}+2 \mathbf{g} \cdot \xi\right) \sqrt{\xi_{x}^{\prime 2}+\xi_{y}^{\prime 2}} d x= \\
& =\left.\dot{x}_{1} \rho\left(\dot{\xi}^{2}+2 \mathrm{v}^{2}+2 \mathbf{g} \cdot \xi\right) \sqrt{\xi_{x}^{\prime 2}+\xi_{y}^{\prime 2}}\right|_{x=x_{1}}+ \\
& +\left.\rho\left(\dot{\xi}^{2}+2 \mathrm{v}^{2}+2 \mathbf{g} \cdot \xi\right) \frac{\left(\xi_{x}^{\prime} \dot{\xi}_{x}+\xi_{y}^{\prime} \dot{\xi}_{y}\right)}{\sqrt{\xi_{x}^{\prime 2}+\xi_{y}^{\prime 2}}}\right|_{x=x_{1}}-
\end{aligned}
$$

$-\int_{x_{0}}^{x_{1}} \rho \dot{\xi}_{x} \frac{\partial}{\partial x}\left[\frac{\xi_{x}^{\prime}\left(\dot{\xi}^{2}+2 \mathrm{v}^{2}+2 \mathbf{g} \cdot \xi\right)}{\sqrt{\xi_{x}^{\prime 2}+\xi_{y}^{\prime 2}}}\right] d x+$

$+\int_{x_{0}}^{x_{1}} \rho(2 \dot{\xi} \cdot \ddot{\xi}+2 \mathbf{g} \cdot \dot{\xi}) \sqrt{\xi_{x}^{\prime 2}+\xi_{y}^{\prime 2}} d x-$

$-\int_{x_{0}}^{x_{1}} \rho \dot{\xi_{y}} \frac{\partial}{\partial x}\left[\frac{\xi_{y}^{\prime}\left(\dot{\xi}^{2}+2 \mathrm{v}^{2}+2 \mathbf{g} \cdot \xi\right)}{\sqrt{\xi_{x}^{\prime 2}+\xi_{y}^{\prime 2}}}\right] d x=0$.

Sum of expressions in equation (13) at arbitrary point of rope $x_{1}=x_{1}\left(\xi_{x}, \xi_{y}\right)$ is a condition of transversality for a $\mathrm{v}$ ariational problem with a movable boundary [9], i.e.

$$
\begin{aligned}
& \left.\dot{x}_{1} \rho\left(\dot{\xi}^{2}+2 \mathrm{v}^{2}+2 \mathbf{g} \cdot \xi\right) \sqrt{\xi_{x}^{\prime 2}+\xi_{y}^{\prime 2}}\right|_{x=x_{1}}+ \\
& +\left.\rho\left(\dot{\xi}^{2}+2 \mathrm{v}^{2}+2 \mathbf{g} \cdot \xi\right) \frac{\left(\xi_{x}^{\prime} \dot{\xi}_{x}+\xi_{y}^{\prime} \dot{\xi}_{y}\right)}{\sqrt{\xi_{x}^{\prime 2}+\xi_{y}^{\prime 2}}}\right|_{x=x_{1}}=0
\end{aligned}
$$

Where does it come from that

$$
\left.\left(\dot{\xi}^{2}+2 \mathrm{v}^{2}+2 \mathbf{g} \cdot \xi\right)\left[\dot{x}_{1} \sqrt{\xi_{x}^{\prime 2}+\xi_{y}^{\prime 2}}+\frac{\left(\xi_{x}^{\prime} \dot{\xi}_{x}+\xi_{y}^{\prime} \dot{\xi}_{y}\right)}{\sqrt{\xi_{x}^{\prime 2}+\xi_{y}^{\prime 2}}}\right]\right]_{x=x_{1}}=0 \mathrm{It}
$$

is clear that the physical sense in this factorial expression can only carry a multiplier in parentheses. Equating it to zero, we get the following condition of transversality

$$
\left(\dot{\xi}^{2}+2 \mathrm{v}^{2}+2 \mathrm{~g} \cdot \boldsymbol{\xi}\right)_{x=x_{1}}=0 .
$$

Note that the equation (14) is nothing but the law of conservation of energy. Indeed, if in the marginal case to assume that the rope is a rigid bar with a dynamic angular variable $\varphi$ that characterizes its deviation from the vertical axis, the parameters included in (14) can be represented as

$$
\begin{aligned}
& \dot{\xi}^{2}=\dot{\xi}_{x}^{2}+\dot{\xi}_{y}^{2}=l_{c}^{2}\left(\frac{d}{d t} \sin \varphi\right)^{2}+l_{c}^{2}\left(\frac{d}{d t} \cos \varphi\right)^{2}=l_{c}^{2} \dot{\varphi}^{2}, \\
& \mathrm{v}=\mathrm{v}_{0}=\text { const } \\
& \mathbf{g} \boldsymbol{\xi}=-g \xi_{y}=-g l_{c} \cos \varphi,
\end{aligned}
$$


where $l_{c}-$ is the center of gravity of the rod, and $x_{1}=l_{c}$. So instead of (14) we immediately get the law of preserving the energy of the rod explicitly

$l_{c}^{2} \dot{\varphi}^{2}-2 g l_{c} \cos \varphi=2 \mathrm{v}_{0}^{2}=$ const.

Thus, given the condition (14) equation (13) is greatly simplified and believing that $\dot{\xi} \cdot \boldsymbol{\tau}=\dot{\xi}_{x} \tau_{x}+\dot{\xi}_{y} \tau_{y}$ and $\mathbf{g} \boldsymbol{\xi} \cdot=-g \dot{\xi}_{y}$, after a simple grouping of the components with $\dot{\xi}_{x}$ and $\dot{\xi}_{y}$ we are finding

$2 \dot{E}=$

$=\int_{x_{0}}^{x_{1}} \rho \dot{\xi}_{x}\left\{\ddot{\xi}_{x} \sqrt{\xi_{x}^{\prime 2}+\xi_{y}^{\prime 2}}-\frac{\partial}{\partial x}\left[\frac{\xi_{x}^{\prime}\left(\dot{\xi}_{x}^{2}+\dot{\xi}_{y}^{2}+2 \mathrm{v}^{2}-2 g \xi_{y}\right)}{\sqrt{\xi_{x}^{\prime 2}+\xi_{y}^{\prime 2}}}\right] d x+\right.$

$+\int_{x_{0}}^{x_{1}} \rho \dot{\xi}_{y}\left\{\left(\ddot{\xi}_{y}+g\right) \sqrt{\xi_{x}^{\prime 2}+\xi_{y}^{\prime 2}}-\frac{\partial}{\partial x}\left[\frac{\xi_{y}^{\prime}\left(\dot{\xi}_{x}^{2}+\dot{\xi}_{y}^{2}+2 \mathrm{v}^{2}-2 g \xi_{y}\right)}{\sqrt{\xi_{x}^{\prime 2}+\xi_{y}^{\prime 2}}}\right] d x=0\right.$.

It should be noted here that the equation (16) does not include energy dissipation. It is obvious that to be taken into account it is necessary to add to (16) the $2 \dot{Q}$ where $\dot{Q}-$ is the dissipative function (also, as it was done, for example, in work [8]). That is, the general equation taking into account the resistance of environment and other braking factors should have the form

$$
\dot{E}+\dot{Q}=0 \text {. }
$$

Due to the independence of the values $\dot{\xi}_{x} \neq 0$ and $\dot{\xi}_{y} \neq 0$ get from eq. (16) the following system of the two main equations

$$
\left\{\begin{array}{l}
\ddot{\xi}_{x} \sqrt{\xi_{x}^{\prime 2}+\xi_{y}^{\prime 2}}-\frac{1}{2} \frac{\partial}{\partial x}\left[\frac{\xi_{x}^{\prime}\left(\dot{\xi}_{x}^{2}+\dot{\xi}_{y}^{2}+2 \mathrm{v}^{2}-2 g \xi_{y}\right)}{\sqrt{\xi_{x}^{\prime 2}+\xi_{y}^{\prime 2}}}\right]=0, \\
\ddot{\xi}_{y} \sqrt{\xi_{x}^{\prime 2}+\xi_{y}^{\prime 2}}-\frac{1}{2} \frac{\partial}{\partial x}\left[\frac{\xi_{y}^{\prime}\left(\dot{\xi}_{x}^{2}+\dot{\xi}_{y}^{2}+2 \mathrm{v}^{2}-2 g \xi_{y}\right)}{\sqrt{\xi_{x}^{\prime 2}+\xi_{y}^{\prime 2}}}\right]= \\
=-g \sqrt{\xi_{x}^{\prime 2}+\xi_{y}^{\prime 2}}
\end{array}\right.
$$

As seen from them, in the marginal case of small oscillations of the heavy rope in the field of gravity from the system of equations (18) after neglecting all quadratic by offsets $\xi_{x}, \xi_{y}$ components, replacing the argument $x$ on $y$, and believing $\xi_{y}=y$ as well as believing that the displacement vector is $\vec{\xi} \approx\left(\xi_{x}, 0\right)$, from the upper equation of the system (18) immediately get the following linear equation

$$
\ddot{\xi}_{x}-\frac{\partial}{\partial y} \xi_{x}^{\prime}\left(\mathrm{v}_{0}^{2}-g y\right)=0,
$$

Which, as it should be, coincides with the given, for example, in [1]. Thus the lower equation is satisfied identically.

In fact, given that $\xi_{y}^{\prime}=1$, it has the following chain of simple transformations

$$
\begin{aligned}
& \ddot{\xi}_{y} \sqrt{\xi_{x}^{\prime 2}+\xi_{y}^{\prime 2}}-g \sqrt{\xi_{x}^{\prime 2}+\xi_{y}^{\prime 2}}- \\
& -\frac{1}{2} \frac{\partial}{\partial y}\left[\frac{\xi_{y}^{\prime}\left(\dot{\xi}_{x}^{2}+\dot{\xi}_{y}^{2}+2 \mathrm{v}_{0}^{2}-2 g y\right)}{\sqrt{\xi_{x}^{\prime 2}+\xi_{y}^{\prime 2}}}\right]=. \\
& =-g-\frac{\partial}{\partial y}\left[\xi_{y}^{\prime}\left(\mathrm{v}_{0}^{2}-g y\right)\right] \equiv 0
\end{aligned}
$$

In the case, if we consider small horizontal oscillations, such as strings, then believing that the vector of bias is $\vec{\xi} \approx\left(0, \xi_{y}\right)$, from the lower equation of the system (18) we will find

$$
\begin{aligned}
& \ddot{\xi}_{y} \sqrt{\xi_{x}^{\prime 2}+\xi_{y}^{\prime 2}}+g \sqrt{\xi_{x}^{\prime 2}+\xi_{y}^{\prime 2}}- \\
& -\frac{1}{2} \frac{\partial}{\partial x}\left[\frac{\xi_{y}^{\prime}\left(\dot{\xi}_{x}^{2}+\dot{\xi}_{y}^{2}+2 \mathrm{v}^{2}-2 g \xi_{y}\right)}{\sqrt{\xi_{x}^{\prime 2}+\xi_{y}^{\prime 2}}}\right] \approx . \\
& \approx \ddot{\xi}_{y}+g-\mathrm{v}_{0}^{2} \xi_{y}^{\prime \prime}=0
\end{aligned}
$$

Here it is taken into account that $\xi_{x}^{\prime}=1$. That is the usual equation of small vibrations of a string in a field of gravity

$$
\ddot{\xi}_{y}-\mathrm{v}_{0}^{2} \xi_{y}^{\prime \prime}=-g
$$

The upper equation is satisfied identically. Indeed, we have for him

$$
\begin{aligned}
& \ddot{\xi}_{x}-\frac{1}{2} \frac{\partial}{\partial x}\left[\frac{\xi_{x}^{\prime}\left(\dot{\xi}_{x}^{2}+\dot{\xi}_{y}^{2}+2 \mathrm{v}^{2}-2 g \xi_{y}\right)}{\sqrt{\xi_{x}^{\prime 2}+\xi_{y}^{\prime 2}}}\right] \approx \\
& \approx \ddot{\xi}_{x}-\mathrm{v}_{0}^{2} \frac{\partial}{\partial x} \xi_{x}^{\prime} \approx 0
\end{aligned}
$$

where it was immediately taken into account that it is only about the transverse oscillations of the 
string, when its longitudinal offsets are absent, that is $\vec{\xi} \approx\left(0, \xi_{y}\right)$.

So, taking into account the equations (4) - (6), as well as the equations of transversality (10) and (14), the system of equations (18) forms a complete closed system of all the basic equations describing the dynamics of arbitrary oscillations of heavy ropes and similar objects, namely

$$
\begin{aligned}
& \left(\ddot{\xi}_{x} \sqrt{\xi_{x}^{\prime 2}+\xi_{y}^{\prime 2}}-\frac{1}{2} \frac{\partial}{\partial x}\left[\frac{\xi_{x}^{\prime}\left(\dot{\xi}_{x}^{2}+\dot{\xi}_{y}^{2}+2 \mathrm{v}^{2}-2 g \xi_{y}\right)}{\sqrt{\xi_{x}^{\prime 2}+\xi_{y}^{\prime 2}}}\right]=0,\right. \\
& \ddot{\xi}_{y} \sqrt{\xi_{x}^{\prime 2}+\xi_{y}^{\prime 2}}-\frac{1}{2} \frac{\partial}{\partial x}\left[\frac{\xi_{y}^{\prime}\left(\dot{\xi}_{x}^{2}+\dot{\xi}_{y}^{2}+2 \mathrm{v}^{2}-2 g \xi_{y}\right)}{\sqrt{\xi_{x}^{\prime 2}+\xi_{y}^{\prime 2}}}\right]= \\
& =-g \sqrt{\xi_{x}^{\prime 2}+\xi_{y}^{\prime 2}}, \\
& \dot{u}=\frac{\mathrm{v}^{2}}{R_{1}}-g \sin \alpha_{1}-k_{1} \frac{u}{\rho}, \\
& \frac{u^{2}}{R_{2}}=g \cos \alpha_{1}-\dot{\mathrm{v}}+\frac{f}{\rho} \text {, } \\
& \mathrm{v}=-R_{1} \dot{\alpha}_{1}, \\
& \left\{k_{1} k_{2} \mid=t\left(\xi_{0}, \xi_{5}\right)=-1\right. \text {, } \\
& \left(\dot{\xi}^{2}+2 \mathrm{v}^{2}+2 \mathbf{g} \cdot \xi\right)_{x=x_{1}}=0, \\
& \frac{1}{R_{1}}=\frac{\frac{d^{2} \xi_{y}}{d \xi_{x}^{2}}}{\left(1+\left(\frac{d \xi_{y}}{d \xi_{x}}\right)^{2}\right)^{\frac{3}{2}},} \\
& \frac{1}{R_{2}}=\frac{\frac{d^{2} \psi}{d \xi_{x}^{2}}}{\left(1+\left(\frac{d \psi}{d \xi_{x}}\right)^{2}\right)^{\frac{3}{2}}} .
\end{aligned}
$$

\section{Analysis of the Equations}

It is clear that it is possible to find the solution of this system of equations in the general form only by numerical methods with corresponding boundary and initial conditions. However, in some individual cases, the resulting common system of nonlinear differential equations, as we have seen above, is still amenable to analysis. As another example, consider this.

Suppose that the rope is absolutely rigid and is a normal rod. In this case, it can be assumed that $\xi_{x}=x=r \sin \varphi, \xi_{y}=y=r \cos \varphi$

where $r-$ is the arbitrary current distance, measured from the anchor point of the bar along its axis, and $\varphi-$ is angle between the bar axis and the axis $y$. So

$$
\operatorname{tg} \varphi=\frac{\xi_{x}}{\xi_{y}}
$$

and corner factor

$$
k_{1}=\frac{d \xi_{y}}{d \xi_{x}}=\operatorname{ctg} \varphi .
$$

Because the length $r$ does not depend on time, then for derivatives in time we have

$$
\dot{\xi}_{x}=r \dot{\varphi} \cos \varphi, \dot{\xi}_{y}=-r \dot{\varphi} \sin \varphi
$$

Further, neglecting friction, and by virtue of the fact that $r=\sqrt{\xi_{x}^{2}+\xi_{y}^{2}}=\sqrt{x^{2}+y^{2}}$ get using the system (21)

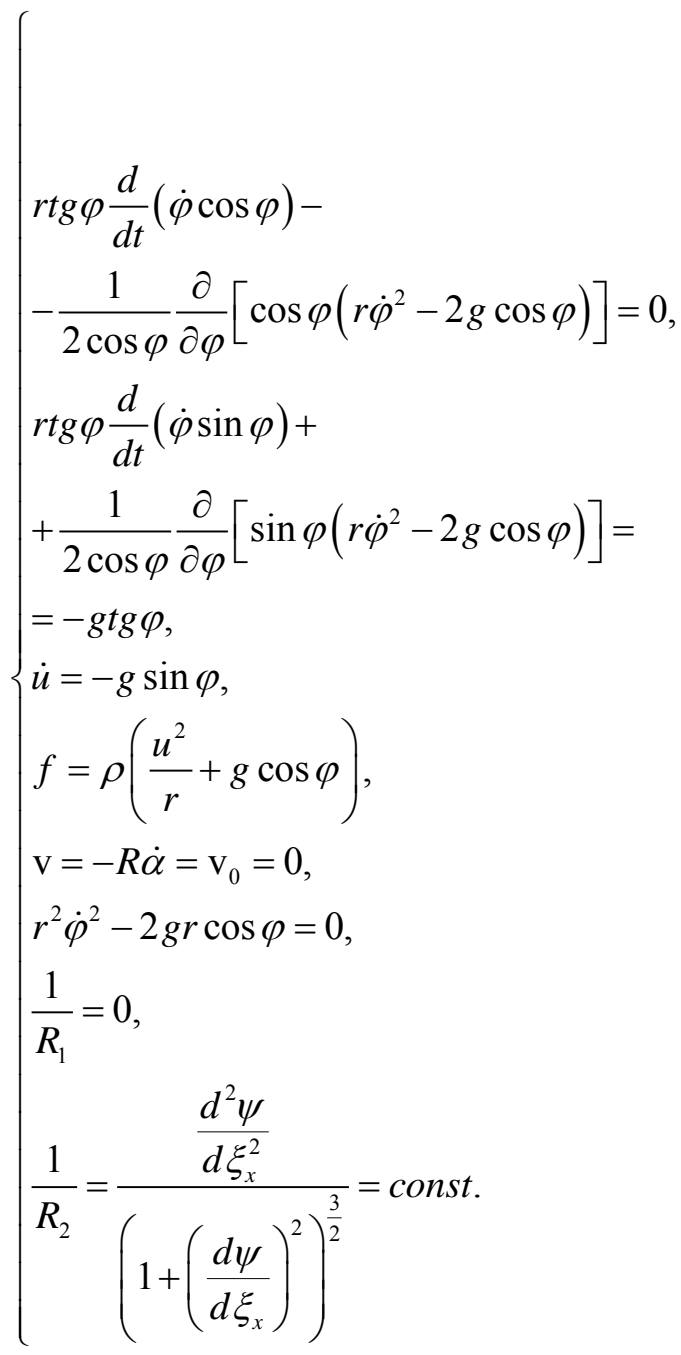


Because $r^{2} \dot{\varphi}^{2}-2 g r \cos \varphi=0$ the first two equations are instantly simplified and given to the view

$$
\begin{aligned}
& \frac{d}{d t}(\dot{\varphi} \cos \varphi)=0, \\
& r \operatorname{tg} \varphi \frac{d}{d t}(\dot{\varphi} \sin \varphi)=-g \operatorname{tg} \varphi .
\end{aligned}
$$

Where, after time differentiation, we will find

$$
\begin{aligned}
& \ddot{\varphi} \cos \varphi-\dot{\varphi}^{2} \sin \varphi=0, \\
& \ddot{\varphi} \sin \varphi+\dot{\varphi}^{2} \cos \varphi=-\frac{g}{r} .
\end{aligned}
$$

Multiplying the upper equation by $\cos \varphi$ and the bottom on $\sin \varphi$, and having folded them, we will get

$\ddot{\varphi}+\frac{g}{r} \sin \varphi=0$.

That is, as it should be, we have got the equation of arbitrary vibrations of a rod, completely equivalent to the third on the top equation of system (22).

As for the boundary and initial conditions for the common system (21), they can be chosen on the basis of the following considerations.

Since one end of the rope is stationary, at this point we have the conditions

$\xi_{x}(0, t)=\xi_{y}(0, t)=0$.

For the rolling end of the rope can use the condition of transversality (14)

$$
\left(\dot{\xi}^{2}+2 \mathrm{v}^{2}+2 \mathbf{g} \cdot \xi\right)_{x=x_{1}}=0 .
$$

As initial conditions it is possible to require execution, for example, such. Let the initial form of the rope be defined as (1), i.e.

$\xi(x, 0)=\mathbf{S}(x)$.

And the initial speed of the rope let it be

$$
\left.\frac{\partial \xi}{\partial t}\right|_{t=0}=\mathbf{V}(x) \text {. }
$$

Thus, it can be argued that the seven conditions (24) - (27) are sufficient for the unambiguous solution of the task. For specific dependencies (26) and (27) numerical integration of equations (21) allows you to find the shape of the rope at any time and for each value of the coordinates $x$.

\section{Main Results}

The system of strong non - linear differential dynamic equations it's find, which describing the behavior of strong dimension objects.

\section{Conclusion}

The merit of the presented work is the description of the method of inducing any dynamic equations with the help of the law of energy conservation, which has not been done before. Therefore, the author's subsequent work will continue the above-mentioned approach in annexing other complex dynamic systems.

In the conclusion of the work we will once again summarize all the main results obtained above.

1. Based on the law of Conservation of energy, obtained a system of nonlinear equations, describing the arbitrary displacement of the rope $\xi(x, t)=\mathbf{i} \xi_{x}(x, t)+\mathbf{j} \xi_{y}(x, t) ;$

2. The physical movement of the surface rope points moving at the speed of $\mathbf{v}$ thanks to oscillatory movements;

3. Obtained a condition of transversality for any point of the rope;

4. It is taken into account the orthogonal movement of the rope with the speed $\mathbf{u}$;

5. On concrete examples the limit physical transitions from the general system of equations (21) to classical tasks are illustrated.

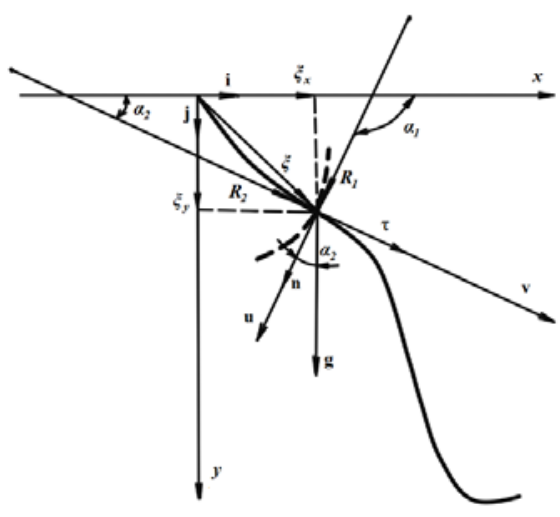

Fig.1. A schematic image of an arbitrary displacements of the rope. All the symbols in the picture are explained in the text of the article. 


\section{References:}

[1] Courant, R. Hilbert, D. Methods of Mathematical Physics., v. 1, Interscience Publ., New York, 1953.

[2] Dacorogna, B., Introduction to the Calculus of Variation. Imperial College Press, London, 2004.

[3] Gladkov S.O. To the theory of nonlinear dynamic equations for the long elastic rod in viscous media. International Journal of Mathematical Models and Methods in Applied Sciences. 2015. V. 9. P. $166-170$.

[4] Gladkov S. O., Bogdanova S. B. Geometric phase transition in the brahistohron problem. Scientists Note Physical Faculty MSU. 2016. №1. 161101-1-5.

[5] Gladkov S. O. On the trajectory of movement of the body entering into the liquid at an arbitrary angle. Scientists Note Physical Faculty Moscow State University. 2016. №4. 164002-1-5.

[6] Gladkov S. O., Bogdanova S. B. Generalized dynamic equations of the flat curvilinear movement of the material body along the groove with regard to friction forces, and their analysis in some private cases. Scientists Note Physical Faculty Moscow State University. 2017. №1. 171101-1-5.

[7] Gladkov S. O., Bogdanova S. B. To the question of analysis of equations of motion of a body on a flat rotating brahistohron. Scientists Note Physical Faculty Moscow state University. 2017. №2. 172101-1-6.

[8] Gladkov S.O. To the question of calculating the stopping time of the cylindrical body rotating in the viscous continuum and the time of hobby of the external cylinder with it. Journal of Technical Physics. 2018. V. 59. N. 3. p. 377.

[9] Gel'fand, I.M., Fomin, S.V. Calculus of Variations. Dover, New York, 2000.

[10] Gurtin, M.E., An Introduction to Continuum Mechanics. Academic Press, New York, 1981.

[11] Hildebrandt, S., Tromba, A., Mathematical and Optimal Form, Scientific American Books, New York, 1985.

[12] Kot, M., A First Course in the Calculus of Variations, American Mathematical Society, Providence, R.I., 2014.

[13] Antman, S.S., Nonlinear Problems of Elasticity, Appl. Math. Sci., vol. 107, Springer-Verlag, New York, 1995.
[14] Ball, J.M., and Mizel, V.J., Onedimensional variational problem whose minimizers do not satisfy the Euler Lagrange equation, Arch. Rat. Mech. Anal. 90 (1985), 325-388.

[15] Schechter, R.S. The Variational Method in Engineering, McGrow-Hill Book Company, 1967.

[16] Van Brunt, B., The Calculus of Variations, Springer, 2004.

[17] Lanczos, C. The Variational Principles of Mechanics, Dover, New York, 1970. 